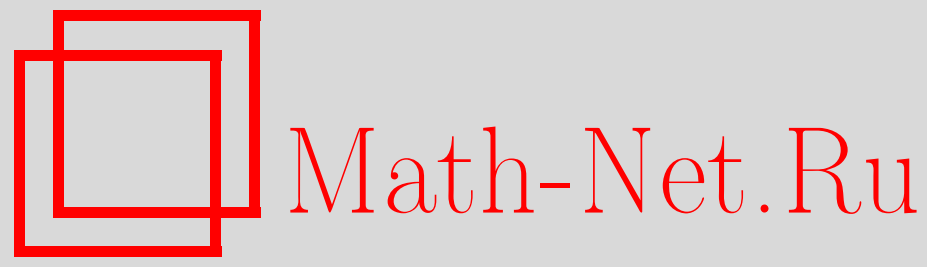

А. И. Овсеевич, Структура аттрактора форм множеств достижимости, Функи. анализ и его прил., 2010, том 44, выпуск 2, 74-81

DOI: https://doi.org/10.4213/faa2985

Использование Общероссийского математического портала MathNet.Ru подразумевает, что вы прочитали и согласны с пользовательским соглашением

http://www . mathnet.ru/rus/agreement

Параметры загрузки:

IP : 52.6 .47 .48

26 апреля 2023 г., 13:06:09

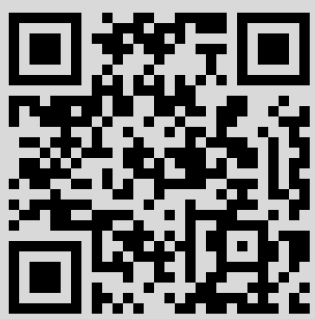


Функционалъный анализ и его приложения

2010, т. 44, вып. 2, с. 74-81

УДК 517.9

\title{
Структура аттрактора форм множеств достижимости
}

\author{
(c) 2010. А. И. ОвСЕЕВич
}

\begin{abstract}
Изучено асимптотическое поведение областей достижимости для линейной автономной импульсной управляемой системы. Основной результат состоит в полном описании множества предельных форм областей достижимости (аттрактора) для случая скалярного управления. Аттрактор оказывается тором, определенным в терминах чисто мнимых собственных значений матрицы системы.
\end{abstract}

Постановка задачи. Рассмотрим линейную автономную конечномерную импульсную управляемую систему

$$
\dot{x}=A x+B u, \quad x \in \mathbb{V}=\mathbb{R}^{n}, x(0)=0,
$$

где $u-$ векторная мера на $\mathbb{R}_{+}$с полной вариацией $\leqslant 1$. Предполагается, что выполнено условие Калмана полной управляемости, т.е. из любой точки можно попасть в любую, двигаясь по траекториям соответствующей системы без ограничений на управление $u$. Обозначим через $D(T)=\{x(T)\}$ множество концов допустимых траекторий системы (1), называемое областъю (множеством) достижимости в момент $T$. Область достижимости - один из центральных объектов теории управления ([1], [2]), имеющий смысл для любых, не только линейных, управляемых систем. В нашем контексте в силу условия Калмана это выпуклое тело - точка пространства Минковского симметричных относительно $0 \in \mathbb{V}$ выпуклых тел. Последнее является полным метрическим пространством относительно метрики Банаха-Мазура

$$
\rho\left(\Omega_{1}, \Omega_{2}\right)=\log \left(t\left(\Omega_{1}, \Omega_{2}\right) t\left(\Omega_{2}, \Omega_{1}\right)\right), \quad \text { где } t\left(\Omega_{1}, \Omega_{2}\right)=\inf \left\{t \geqslant 1: t \Omega_{1} \supset \Omega_{2}\right\} .
$$

При сопоставлении выпуклому телу $\Omega$ его опорной функции

$$
H_{\Omega}(\xi)=\sup _{x \in \Omega}\langle x, \xi\rangle
$$

пространство Минковского отождествляется с множеством банаховых норм на двойственном пространстве $\mathbb{V}^{*}$. Множество банаховых норм на исходном пространстве $\mathbb{V}$ также естественно отождествляется с пространством Минковского при сопоставлении норме соответствующего единичного шара.

Через Sh $D(T)$ обозначим форму тела $D(T)$, т. е. орбиту $\{g D(T): g \in \mathrm{GL}(\mathbb{V})\}$ точки $D(T)$ в пространстве Минковского под действием полной линейной группы. Это элемент пространства $\mathbb{S}$ форм симметричных выпуклых тел, которое является компактным метрическим пространством с факторметрикой БанахаМазура. Факторметрика здесь понимается стандартным образом: $\rho\left(\operatorname{Sh} \Omega_{1}, \operatorname{Sh} \Omega_{2}\right)$ $=\inf \left\{\rho\left(g_{1} \Omega_{1}, g_{2} \Omega_{2}\right), g_{i} \in \mathrm{GL}(\mathbb{V})\right\}$. Пространство $\mathbb{S}$ можно естественным образом трактовать как множество банаховых норм на $\mathbb{V}$ или $\mathbb{V}^{*}$ с точностъю до линейного изоморфизма. Компактность пространства $\mathbb{S}$ легко получается, например, из классической теоремы Джона [5], утверждающей, что любое выпуклое симметричное тело находится на конечном $\left(\leqslant \frac{1}{2} \log n\right)$ расстоянии Банаха-Мазура 
от некоторого эллипсоида, и критерия компактности Асколи-Арцела в применении к опорным функциям.

Через $\mathscr{A}$ обозначим аттрактор - совокупность всех $\omega$-предельных точек кривой $T \mapsto \operatorname{Sh} D(T)$ :

$$
\mathscr{A}=\left\{\lim _{T_{k} \rightarrow+\infty} \operatorname{Sh} D\left(T_{k}\right)\right\} \subset \mathbb{S} .
$$

Структура аттрактора $\mathscr{A}$ в значительной (но не полной) мере известна [3]. Для ее описания необходимо ввести некоторые обозначения. Рассмотрим каноническое разложение

$$
A=A_{+} \oplus A_{0} \oplus A_{-}
$$

матрицы $A$ системы $(1)$ на неустойчивую, нейтральную и устойчивую компоненты в соответствии со знаком действительных частей ее собственных значений. Разложение (2) порождает разложение фазового пространства $\mathbb{V}$ в прямую сумму

$$
\mathbb{V}=\mathbb{V}_{+} \oplus \mathbb{V}_{0} \oplus \mathbb{V}_{-},
$$

которое позволяет записывать векторы $x \in \mathbb{V}$ в виде троек $\left(x_{+}, x_{0}, x_{-}\right)$, где $x_{i} \in \mathbb{V}_{i}$. Определим $\mathbb{V}_{i j}$, где $i, j \in\{+, 0,-\}$, как сумму $\mathbb{V}_{i} \oplus \mathbb{V}_{j}$.

Рассмотрим разложение Жордана нейтральной компоненты матрицы $A$ :

$$
A_{0}=D+N, \quad D N=N D,
$$

где $D$ - диагонализуемая, а $N$ - нильпотентная матрицы. Существует (неоднозначно определенная) матрица $F_{\infty}=F_{\infty}(N): \mathbb{V}_{0} \rightarrow \mathbb{V}_{0}$ со следующими свойствами: $F_{\infty} N=0, F_{\infty} D=D F_{\infty}$, факторотображение $\bar{F}_{\infty}: \overline{\mathbb{V}}_{0} \rightarrow \overline{\mathbb{V}}_{0}$, где $\overline{\mathbb{V}}_{0}=\mathbb{V}_{0} / N \mathbb{V}_{0}$, тождественное.

Для выпуклых компактов $\Omega_{i}$ будем обозначать выпуклую оболочку $\Omega$ их объединения знаком соединения (джойна): $\Omega=\Omega_{1} * \Omega_{2}$.

Определим тор $\mathscr{T} \subset \mathrm{GL}\left(\mathbb{V}_{0}\right)$ как замыкание однопараметрической подгруппы $\left\{e^{D t}\right\} \subset \mathrm{GL}\left(\mathbb{V}_{0}\right)$.

Основное утверждение о структуре аттрактора состоит в следующем [3].

Теорема 1. Аттрактор $\mathscr{A}$ представляет собой образ тора Я при некотором непрерывном отображсении $\Phi$, а кривая $T \mapsto \operatorname{Sh} D(T)$ в пространстве борм асимптотически близка $к$ образу $T \mapsto \Phi(\gamma(T))$ прямолинейной обмотки $\gamma(T)=e^{D T}$ тора $\mathscr{T}$. Более точно, $\Phi(\mathbf{t})=\operatorname{Sh} \Omega(\mathbf{t})$, где

$$
\Omega(\mathbf{t})=\left(\mathbf{t} \Omega_{+0}\right) * \Omega_{0} * \Omega_{-0} .
$$

Здесъ $\Omega_{\alpha} \subset \mathbb{V}_{\alpha}, \alpha \in\{+0,0,-0\}$, - некоторые не зависящие от времени въпуклье компакты.

Говоря еще точнее, опорные функции множеств $\Omega_{\alpha}$ задаются явными формулами

$$
\begin{aligned}
H_{\Omega_{-0}}(\xi) & =\sup \left|B^{*} F_{\infty}^{*} e^{D^{*} t} \xi_{0}+B^{*} e^{A_{-}^{*} t} \xi_{-}\right|, & & t \in[0, \infty), \\
H_{\Omega_{+0}}(\xi) & =\sup \mid B^{*} F_{\infty}^{*} e^{-D^{*} s} e^{N^{*}} \xi_{0}+B^{*} e^{-A_{+}^{*} \xi_{+} \mid,} & & t \in[0, \infty), \\
H_{\Omega_{0}}(\xi) & =\sup \left|B^{*} F_{\infty}^{*} \mathbf{t}^{*} e^{N^{*}} \xi_{0}\right|, & & \mathbf{t} \in \mathscr{T}, \tau \in J=[0,1] .
\end{aligned}
$$

Нас интересует полное описание аттрактора, т. е., в свете теоремы 1, вопрос о том, когда предельные формы $\mathrm{Sh} \Omega(\mathbf{t})$ совпадают при разных t. Аналогичная проблема изучалась в [4] для периодических линейных систем с ограниченными управлениями. 
Здесь мы рассмотрим только случай скалярного управления. Кроме того, считаем, что все компоненты $\mathbb{V}_{i}, i= \pm, 0$, ненулевые, поскольку в противном случае все предельные формы совпадают по тривиальным причинам и аттрактор сводится к точке. Например, в случае $\mathbb{V}_{-}=0$ формула (3) сводится к равенству $\Omega(\mathbf{t})=\left(\mathbf{t} \Omega_{+0}\right) * \Omega_{0}=\mathbf{t}\left(\Omega_{+0} * \Omega_{0}\right)$, поскольку множество $\Omega_{0}$ инвариантно относительно тора $\mathscr{T}$. Следовательно, $\mathrm{Sh} \Omega(\mathbf{t})=\mathrm{Sh} \Omega_{+0} * \Omega_{0}$ не зависит от $\mathbf{t} \in \mathscr{T}$.

Для различения предельных форм $\mathrm{Sh} \Omega(\mathbf{t})$ при разных $\mathbf{t}$ требуются инварианты - свойства множеств $\Omega(\mathbf{t})$, сохраняющиеся под действием $\mathrm{GL}(\mathbb{V})$. Идея состоит в том, чтобы изучить множество крайних точек в $\Omega(\mathbf{t})$ и выделить в нем некоторые особенно замечательные кривые, которые и дают нужные инварианты.

\section{Вспомогательный результат.}

Теорема 2. Пусть $S \in \mathrm{GL}(\mathbb{V})$ отображсает кривую $\gamma=\left\{e^{A t} b, t \geqslant 0\right\}$ в аналогичную кривую $\gamma^{\prime}=\left\{e^{A^{\prime} t} b^{\prime}, t \geqslant 0\right\}$, m.e. $S e^{A t} b=e^{A^{\prime} \phi(t)} b^{\prime}$, где $\phi(0)=0$. Предположим, что выполнено условие Калмана: кривая $\gamma$ линейно порождает все пространство $\mathbb{V}$. Тогда $S A S^{-1}=\lambda A^{\prime}$, где $\lambda$ - положительная константа.

Иными словами, если одна фазовая кривая системы $\dot{x}=A x$ переводится линейным преобразованием $S$ в фазовую кривую системы $\dot{x}=A^{\prime} x$, то $S$ переводит весь фазовый портрет в фазовый портрет.

В самом деле, положим $A^{\prime \prime}=S A S^{-1}$. Тогда $e^{A^{\prime \prime} t} b^{\prime}=e^{A^{\prime} \phi(t)} b^{\prime}$. При этом $\phi$ - бесконечно гладкая и даже аналитическая неотрицательная возрастающая функция на $[0, \infty)$. Дифференцируя по $t$, получим

$$
\left(A^{\prime \prime}-\phi(t)^{\prime} A^{\prime}\right) e^{A^{\prime \prime} t} b^{\prime} \equiv 0 .
$$

Отсюда следует, что $\operatorname{det}\left(A^{\prime \prime}-\phi(t)^{\prime} A^{\prime}\right) \equiv 0$. Однако уравнение

$$
\operatorname{det}\left(A^{\prime \prime}-\lambda A^{\prime}\right)=0
$$

имеет лишь конечное число решений $\lambda$. В частности, любое непрерывное семейство корней постоянно. Следовательно, $\phi(t)^{\prime}$ есть константа $\lambda$ и, значит,

$$
\left(A^{\prime \prime}-\lambda A^{\prime}\right) x=0
$$

при $x \in \gamma^{\prime}$. Но поскольку кривая $\gamma^{\prime}$ линейно порождает все пространство, получается, что $A^{\prime \prime}-\lambda A^{\prime}=0$.

Выделенные кривые. Откуда берутся кривые в нашей исходной задаче?

Пусть управление скалярное. Тогда систему (1) можно записать в виде

$$
\dot{x}=A x+b u, \quad x(0)=0,
$$

где $b$ - вектор, а $u-\left(\right.$ вещественная) мера на $\mathbb{R}_{+}$с полной вариацией $\leqslant 1$; условие Калмана означает, что кривая $e^{A t} b$ линейно порождает все фазовое пространство. При этом $\Omega_{-0}$ - выпуклая оболочка «кривой»

$$
\gamma_{-0}(t)= \pm\left(e^{A_{-} t} b_{-}+e^{D t} b_{0}^{-}\right), \quad b_{0}^{-}=F_{\infty} b_{0},
$$

$\Omega_{+0}$ - выпуклая оболочка «кривой»

$$
\gamma_{+0}(t)= \pm\left(e^{-A_{+} t} b_{+}+e^{-D t} b_{0}^{+}\right), \quad b_{0}^{+}=e^{N} F_{\infty} b_{0},
$$

$\Omega_{0}$ - выпуклая оболочка множества $\gamma_{0}$ точек вида

$$
\pm e^{D t} e^{\tau N} b_{00}, \quad \text { где } b_{00}=b_{0}^{-}=F_{\infty} b_{0}, t \in \mathbb{R}, \tau \in I=[0,1] .
$$


Слово «кривая» в применении к $\gamma_{-0}$ и $\gamma_{+0}$ поставлено в кавычки, поскольку каждое из этих множеств на самом деле есть объединение двух кривых, причем эти кривые получаются друг из друга с помощью центральной симметрии. В дальнейшем будем эти кавычки опускать. Впрочем, $\gamma_{-0}$ и $\gamma_{+0}$ можно рассматривать как настоящие кривые, но в проективных пространствах $\mathbb{P}\left(\mathbb{V}_{ \pm 0}\right)$.

Восстановление кривых $\gamma_{ \pm 0}$ по телу $\Omega$. Утверждается, что на каждой из кривых $\gamma_{-0}$ и $\gamma_{+0}$ имеются плотные подмножества, состоящие из выступающих точек множества

$$
\Omega=\Omega_{+0} * \Omega_{0} * \Omega_{-0} .
$$

Напомним, что если $\Omega$ - выпуклый компакт, то точка $x \in \Omega$ называется выступающей, если для некоторого двойственного вектора $\xi$ функция

$$
\Omega \ni y \mapsto\langle y, \xi\rangle
$$

имеет в точке $x$ строгий максимум. Разумеется, множество $\Gamma(\Omega)$ выступающих точек тела $\Omega$ является его линейным инвариантом в том смысле, что $\Gamma(g \Omega)=g \Gamma(\Omega)$ для $g \in \mathrm{GL}(\mathbb{V})$. Инвариантом является и замыкание множества выступающих точек.

Поскольку $\Omega$ является выпуклой оболочкой объединения

$$
\gamma=\gamma_{-0} \cup \gamma_{+0} \cup \gamma_{0}
$$

то вне этого объединения выступающих точек нет.

Рассмотрим сначала множество $\gamma_{0}$. Пусть $t_{*} \geqslant 0, \tau_{*} \in I$ и

$$
\gamma_{0}\left(t_{*}, \tau_{*}\right)=e^{D t_{*}} e^{\tau_{*} N} b_{00}
$$

- соответствующая точка в $\gamma_{0}$. Функция

$$
h_{0}=h_{\xi_{0}}:(t, \tau) \mapsto\left\langle e^{D t} e^{\tau N} b_{00}, \xi\right\rangle
$$

имеет вид $\operatorname{Re} \sum_{\omega} e^{i \omega t} p_{\omega, \xi}(\tau)$, где суммирование проводится по неотрицательным частотам $\omega \geqslant 0$, причем полином $p_{\omega, \xi}(\tau)$ является комплексным при $\omega \neq 0$ и вещественным при $\omega=0$. Более того, варьируя $\xi=\xi_{0}$, можно сделать полиномы $p_{\omega, \xi}$ произвольными в рамках единственного ограничения - степень полинома $p_{\omega, \xi}$ меньше размера соответствующей жордановой клетки матрицы $N$.

Запишем $\operatorname{Re} \sum_{\omega} e^{i \omega t} p_{\omega, \xi}(\tau)$ в виде $\operatorname{Re} \sum_{\omega} e^{i \omega\left(t-t_{*}\right)} P_{\omega, \xi}(\tau)$, где на полиномы $P_{\omega, \xi}$ наложены те же самые ограничения, что и на $p_{\omega, \xi}$. Поэтому если $P_{\omega, \xi}\left(\tau_{*}\right)=$ $\max _{\tau \in I}\left|P_{\omega, \xi}(\tau)\right|$ для всех индексов $\omega$, то $\left(t_{*}, \tau_{*}\right)$ - точка максимума функции $h_{0}$, причем этот максимум положительный и строгий по $t$. Более того, выполнено стандартное достаточное условие строгости максимума:

$$
\frac{\partial^{2} h_{0}}{\partial t^{2}}\left(t_{*}, \tau_{*}\right)<0 .
$$

Добиться того, чтобы максимум был строгим и по $\tau$, не всегда возможно: для этого нужно, чтобы степень хотя бы одного из полиномов $p_{\omega, \xi}$ была больше единицы или, что эквивалентно, чтобы размер одной из жордановых клеток матрицы $N$ был не меньше трех. В этом случае все точки $\gamma_{0}\left(t_{*}, \tau_{*}\right)$, где $t_{*} \geqslant 0$, $\tau_{*} \in I$, выступающие. Если размеры всех жордановых клеток матрицы $N$ меньше трех, то из предыдущих рассуждений вытекает, что выступающие точки это все точки вида $\gamma_{0}\left(t_{*}, 0\right)$ или $\gamma_{0}\left(t_{*}, 1\right)$. Соответственно замыкание $\Gamma_{0}$ множества выступающих точек вида $\gamma_{0}(t, \tau)$ - это либо множество $M=\left\{\mathbf{x} e^{\tau N} b_{00}\right.$, 
$\mathbf{x} \in \mathscr{T}, \tau \in I\}$, либо объединение множеств $M_{ \pm}=\left\{\mathbf{x} b_{0}^{ \pm}, \mathbf{x} \in \mathscr{T}\right\}$, которое также можно описать как множество точек вида $\mathbf{x} e^{\tau N} b_{00}$, где $\tau \in \partial I=\{0,1\}$. В последнем случае возможно, что $N=0$, и тогда множества $M_{+}$и $M_{-}$точек вида $\mathbf{x} e^{\tau N} b_{00}$ при $\tau=0$ и $\tau=1$ совпадают друг с другом и с $M$. «Неблагоприятный» случай - это когда максимальный размер жордановых клеток матрицы $N$ равен двум. Тогда множество $M$ действительно не совпадает с $\Gamma_{0}$ и получается из $\Gamma_{0}$ путем присоединения к $\Gamma_{0}$ прямолинейных отрезков $I(\mathbf{x})$, соединяющих точки $\mathbf{x} b_{0}^{+}$и $\mathbf{x} b_{0}^{-}$.

Покажем теперь, что точку $\gamma_{-0}\left(t_{*}\right)=e^{A_{-} t_{*}} b_{-}+e^{D t_{*}} F_{\infty} b_{0}$ можно сколь угодно хорошо приблизить выступающими точками на кривой $\gamma_{-0}$. Функцию $h_{-0}(t)=$ $\left\langle\gamma_{-0}(t), \xi\right\rangle=\left\langle e^{A_{-} t} b_{-}, \xi_{-}\right\rangle+h_{\xi_{0}}(t, 0)$ при малом двойственном векторе $\xi_{-}$можно рассматривать как малое возмущение функции

$$
h^{0}(t)=h_{\xi_{0}}(t, 0),
$$

которая имеет положительный строгий максимум в точке $t_{*}$. Пусть вектор $\xi_{-}$ не только маленький, но еще и такой, что

$$
\left\langle e^{A_{-} t_{*}} b_{-}, \xi_{-}\right\rangle>0 \text {. }
$$

Тогда, во-первых, максимум функции $\Omega \ni y \mapsto\langle y, \xi\rangle$, где $\xi=\left(\xi_{-}, \xi_{0}, 0\right)$, достигается на кривой $\gamma_{-0}$, поскольку на $\gamma_{+0}$ и $\gamma_{0}$ ее значения меньше в силу неравенства $h_{-0}\left(t_{*}\right)>h^{0}\left(t_{*}\right)=\max h^{0}(t)$. Во-вторых, если вектор $\xi_{-}$достаточно мал, то этот максимум достигается в единственной точке вблизи точки $t_{*}$, причем в точке максимума выполнено условие невырожденности $\partial^{2} h_{-0} / \partial t^{2}<0$. В частности, этот максимум строгий, и, таким образом, сколь угодно близко к $\gamma_{-0}\left(t_{*}\right)$ на $\gamma_{-0}$ имеется выступающая точка.

Совершенно аналогичное рассуждение показывает, что на кривой $\gamma_{+0}$ имеется плотное множество выступающих точек для тела $\Omega$.

Выводы. Первый вывод из этих рассмотрений таков: по телу $\Omega$ можно однозначно восстановить кривые $\gamma_{ \pm 0}$. В самом деле, по телу $\Omega$ можно однозначно восстановить замыкание $\bar{\Gamma}=\bar{\gamma}$ множества выступающих точек, которое, как мы выяснили, имеет вид $\bar{\Gamma}=\gamma_{-0} \cup \Gamma_{0} \cup \gamma_{+0}$. Геометрически мы имеем два одномерных уса $\gamma_{ \pm 0}$, наматывающихся на многообразие (возможно, с краем) $\Gamma_{0}$. Компоненты разложения определяются по $\bar{\Gamma}$ однозначно. Действительно, внутренняя точка кривой $\gamma_{ \pm 0}$ выделяется из прочих точек множества $\bar{\Gamma}$ тем, что если ее удалить из $\bar{\Gamma}$, то образуется дополнительная связная компонента.

Точно такие же рассуждения позволяют восстановить по телу $\Omega(\mathbf{t})$ пару «кривых» $\left\{\mathbf{t} \gamma_{+0}, \gamma_{-0}\right\}$.

Второй вывод состоит в том, что по телу $\Omega$ можно однозначно восстановить множество $M=\left\{\mathbf{x} e^{\tau N} b_{00}, \mathbf{x} \in \mathscr{T}, \tau \in I\right\}$. В самом деле, множество $\Gamma_{0}=\bar{\gamma} \backslash\left(\gamma_{-0} \cup \gamma_{+0}\right)$ восстанавливается по $\Omega$ однозначно. Пусть осуществляется «благоприятный» случай, когда либо $N=0$, либо у $N$ имеется жорданова клетка размера $\geqslant 3$. Тогда $M=\Gamma_{0}$, и тем самым $M$ восстанавливается по $\Omega$ однозначно.

Пусть реализуется «неблагоприятный» случай, когда $N \neq 0$, а все жордановы клетки имеют размеры $\leqslant 2$. Рассмотрим подпространство $\mathbb{V}_{\infty}=\mathbb{V}_{+} \oplus$ $F_{\infty} \mathbb{V}_{0} \oplus \mathbb{V}_{-} \subset \mathbb{V}$. Отметим, что вектор $b_{-0}=\left(b_{-}, b_{0}^{-}\right)$в обозначениях (4) лежит 
в $\mathbb{V}_{\infty}$, а оператор $\widetilde{A}=A_{+} \oplus D \oplus A_{-}$действует в этом пространстве. Инвариантное подпространство $\mathbb{V}_{\infty-0}=F_{\infty} \mathbb{V}_{0} \oplus \mathbb{V}_{-}$- линейная оболочка кривой $\gamma_{-0} \subset \mathbb{V}_{\infty}$. Теперь применим вспомогательную теорему 2 к кривой $\gamma_{-0}$, рассматриваемой как множество. Получим, что параметризованная кривая $\gamma_{-0}(t)$ восстанавливается по $\Omega$ однозначно с точностью до линейной замены параметра $t \mapsto \lambda_{-} t, \lambda_{-}>0$. Точно так же восстанавливается параметризованная кривая $\gamma_{+0}(t) \in \mathbb{V}_{+} \oplus e^{N} F_{\infty} \mathbb{V}_{0}$ с точностью до линейной замены $t \mapsto \lambda_{+} t, \lambda_{+}>0$. Утверждается, что $\lambda_{+}=\lambda_{-}$. Действительно, замыкания кривых $\gamma_{ \pm 0}(t)-$ главные однородные пространства одного и того же тора $\mathscr{T}$, и мы получаем автоморфизм тора, продолжающий автоморфизм $\exp (t D) \mapsto \exp \left(\frac{\lambda_{+}}{\lambda_{-}} t D\right)$ порождающей однопараметрической группы. Но такое продолжение существует, только если $\lambda_{+} / \lambda_{-}=1$. Следовательно, соответствие $\mathbf{x} b_{0}^{+} \mapsto \mathbf{x} b_{0}^{-}$между множествами $M_{+}$и $M_{-}$определено телом $\Omega$ однозначно. Во избежание недоразумений отметим, что восстановление канонического соответствия между множествами $M_{+}$и $M_{-}$происходит не только в «неблагоприятном» случае. Однако только в этом случае множество $M$ получается из $M_{+}$и $M_{-}$путем присоединения прямолинейных отрезков $I(\mathbf{x})$, соединяющих соответствующие точки, и потому также определяется однозначно.

Рассмотрим «аналитическое продолжение»

$$
\widetilde{M}=\left\{\mathbf{x} e^{\tau N} b_{00},(\mathbf{x}, \tau) \in \mathscr{T} \times \mathbb{R}\right\}
$$

множества $M$. Это главное однородное пространство группы $\mathscr{T} \times \mathbb{R}$, которое как множество определяется по телу $\Omega$ однозначно. Покажем, что естественная проекция $\widetilde{M} \rightarrow M_{-}$, задаваемая формулой $\mathbf{x} e^{\tau N} b_{00} \mapsto \mathbf{x} b_{00}$, определяется по телу $\Omega$ однозначно. В самом деле, она задается полиномиальным (в естественных матричных координатах) отображением $\pi: \mathscr{T} \times \mathbb{R} \rightarrow \mathscr{T}$, которое обладает свойством $\pi(\mathbf{x}, 0)=\mathbf{x}$. Однако такое отображение единственно: не существует непостоянных полиномиальных отображений $\mathbb{R} \rightarrow \mathscr{T}$.

Следовательно, слои отображения $\pi$, которые являются орбитами однопараметрической группы $\{\exp (N \tau)\}$, определяются по телу $\Omega$ однозначно. Кроме того, отображение $\exp (N): M_{-} \rightarrow M_{+}$, как мы уже выяснили, восстанавливается по телу $\Omega$. Следовательно, действие группы $\exp (N \tau)$ определяется по телу $\Omega$ однозначно с точностью до знака $\tau$ (обращение соответствует перестановке $M_{-}$и $\left.M_{+}\right)$.

Плоды просвещения. В частности, если $\mathrm{Sh} \Omega\left(\mathbf{t}^{\prime}\right)=\mathrm{Sh} \Omega\left(\mathbf{t}^{\prime \prime}\right)$, то отсюда следует, что имеется матрица $S \in \mathrm{GL}(\mathbb{V})$, которая переводит пару кривых $\left\{\mathbf{t}^{\prime} \gamma_{+0}, \gamma_{-0}\right\}$ в аналогичную пару $\left\{\mathbf{t}^{\prime \prime} \gamma_{+0}, \gamma_{-0}\right\}$ и, кроме того, коммутирует или антикоммутирует с матрицей $N$.

При этом могут представиться два случая: либо

$$
S \mathbf{t}^{\prime} \gamma_{+0}=\mathbf{t}^{\prime \prime} \gamma_{+0}, \quad S \gamma_{-0}=\gamma_{-0}, \quad S N=N S,
$$

либо

$$
S \mathbf{t}^{\prime} \gamma_{+0}=\gamma_{-0}, \quad S \gamma_{-0}=\mathbf{t}^{\prime \prime} \gamma_{+0}, \quad S N=-N S .
$$

Из теоремы 2 получаем, что в первом случае

$$
S \widetilde{A} S^{-1}=\lambda \widetilde{A} \quad \text { и } \quad S \mathbf{t}^{\prime} b_{+0}= \pm \mathbf{t}^{\prime \prime} b_{+0}, \quad S b_{-0}= \pm b_{-0},
$$


а во втором

$$
S \widetilde{A} S^{-1}=-\lambda \widetilde{A} \quad \text { и } \quad S \mathbf{t}^{\prime} b_{+0}= \pm b_{-0}, \quad S b_{-0}= \pm \mathbf{t}^{\prime \prime} b_{+0} ，
$$

где $b_{+0}=\left(b_{+}, b_{0}^{+}\right), b_{-0}=\left(b_{-}, b_{0}^{-}\right)$в обозначениях $(4),(5), \lambda$ - положительная константа, а оператор $\widetilde{A}=A_{+} \oplus D \oplus A_{-}$действует в пространстве $\mathbb{V}_{\infty}=$ $\mathbb{V}_{+} \oplus F_{\infty} \mathbb{V}_{0} \oplus \mathbb{V}_{-}$. Для определения константы $\lambda$ используем следующее простое соображение: спектр $\operatorname{Spec}\left(S \widetilde{A} S^{-1}\right)$ матрицы $S \widetilde{A} S^{-1}$ совпадает со спектром $\operatorname{Spec}(\widetilde{A}), \operatorname{a} \operatorname{Spec}(\lambda \widetilde{A})=\lambda \operatorname{Spec}(\widetilde{A})$. Следовательно, $\operatorname{Spec}(\widetilde{A})=\lambda \operatorname{Spec}(\widetilde{A}) \cdot \operatorname{Taк~как~}$ спектр - конечное множество, а $\lambda>0$, то $\lambda=1$. Заметим, что первый случай, $(7)$, сводится ко второму, (6), если рассмотреть преобразование $S^{2}$ вместо $S$ и учесть, что $S \mathbf{t}=\mathbf{t}^{-1} S$ при $\mathbf{t} \in \mathscr{T}$. Последнее вытекает из первого соотношения (9) с учетом равенства $\lambda=1$. Конечно, второй случай следует рассматривать как исключительный, поскольку для его возникновения нужно, например, чтобы спектр матрицы $A$ был симметричным: $\operatorname{Spec}(A)=-\operatorname{Spec}(A)$.

Из последних двух соотношений (8) получим, что $\mathbf{t}^{\prime} b_{0}^{-}= \pm \mathbf{t}^{\prime \prime} b_{0}^{-}$.

В самом деле, из последнего соотношения (8) имеем $S b_{0}^{-}= \pm b_{0}^{-}$, а из предпоследнего $S \mathbf{t}^{\prime} e^{N} b_{0}^{-}= \pm \mathbf{t}^{\prime \prime} e^{N} b_{0}^{-}$. В силу (6) и (8) преобразование $S$ коммутирует с $N$ и с тором $\mathscr{T}$. Следовательно, $\mathbf{t}^{\prime} b_{0}^{-}= \pm S \mathbf{t}^{\prime} b_{0}^{-}= \pm \mathbf{t}^{\prime \prime} b_{0}^{-}$.

Поскольку действие тора $\mathscr{T}$ коммутирует с $N$, а векторы вида $N^{k} \mathbf{t} b_{0}^{-}$, где $\mathbf{t} \in \mathscr{T}$, порождают все пространство $\mathbb{V}_{0}$, мы получаем, что $\mathbf{t}^{\prime}= \pm \mathbf{t}^{\prime \prime}$.

Следовательно, множества $\Omega\left(\mathbf{t}^{\prime}\right)$ и $\Omega\left(\mathbf{t}^{\prime \prime}\right)$ имеют одинаковую форму, только если $\mathbf{t}^{\prime}= \pm \mathbf{t}^{\prime \prime}$. Ясно, что если $\mathbf{t}^{\prime}= \pm \mathbf{t}^{\prime \prime}$, то отображение

$$
S:\left(x_{+}, x_{0}, x_{-}\right) \mapsto\left(x_{+},-x_{0},-x_{-}\right)
$$

переводит $\Omega\left(\mathbf{t}^{\prime}\right)$ в $\Omega\left(\mathbf{t}^{\prime \prime}\right)$, так что условие $\mathbf{t}^{\prime}= \pm \mathbf{t}^{\prime \prime}$ является не только необходимым, но и достаточным для того, чтобы $\operatorname{Sh} \Omega\left(\mathbf{t}^{\prime}\right)=\operatorname{Sh} \Omega\left(\mathbf{t}^{\prime \prime}\right)$. В частности, если матрица -1 из группы $\mathrm{GL}\left(\mathbb{V}_{0}\right)$ не принадлежит $\mathscr{T}$, то аттрактор совпадает с тором.

Нетрудно дать критерий того, что $-1 \in \mathscr{T}$. А именно, нужно рассмотреть все целочисленные (резонансные) соотношения

$$
\sum_{k=1}^{d} m_{k} \omega_{k}=0
$$

между «частотами» $\omega_{k}$ или, что то же самое, собственными значениями $\lambda_{k}=$ $i \omega_{k}$ матрицы $A_{0}$. Тогда критерий состоит в том, что для любого соотношения (10) число $\sum m_{k}$ четное.

Вообще, пусть $z=\operatorname{diag}\left(z_{1}, \ldots, z_{d}\right)$ - диагональная матрица в том же базисе, в котором диагонализуется матрица $D=i \operatorname{diag}\left(\omega_{1}, \ldots, \omega_{d}\right)$. Тогда $z \in \mathscr{T}$ в том и только в том случае, когда

$$
\left|z_{k}\right|=1 \text { для всех } k \text { и } \prod_{k=1}^{d} z_{k}^{m_{k}}=1 \text { для любого соотношения (10). }
$$

Ясно, что при $z=-1$ мы приходим к условию $\prod(-1)^{m_{k}}=(-1)^{\sum m_{k}}=1$ для любого соотношения (10), а это и означает, что число $\sum m_{k}$ четное. Критерий (11) - это классическая теорема Кронекера, которая утверждает, что 
вектор $\left(z_{1}, \ldots, z_{d}\right) \in T^{d}$ можно сколь угодно хорошо приблизить вектором вида $\left(e^{i \omega_{1} t}, \ldots, e^{i \omega_{d} t}\right) \in T^{d}$, если (и только если) выполнено условие (11). (Здесь $T=\{w \in \mathbb{C}:|w|=1\}$.

[Теорема Кронекера вытекает «из общих принципов», а именно из теории двойственности локально компактных абелевых групп. Действительно, замыкание $\mathscr{T}$ однопараметрической подгруппы $\left\{\left(e^{i \omega_{1} t}, \ldots, e^{i \omega_{d} t}\right)\right\} \subset T^{d}$ должно совпадать с подмножеством в $T^{d}$, на котором обращаются в единицу все характеры $T^{d}$, обращающиеся в единицу на однопараметрической подгруппе. Но это и есть теорема Кронекера.]

В случае «общего положения» нетривиальных резонансов (10) вообще нет и, в частности, $-1 \in \mathscr{T}$. Но если резонансы присутствуют, то случай $-1 \notin \mathscr{T}$ встречается чаще, чем противоположный.

Итак, основной результат - следующий.

Теорема 3. Пусть в линейной системе (1) управление и скалярное, а все пространства $\mathbb{V}_{i}, i \in\{+, 0,-\}$, ненулевые. Тогда аттрактор $\mathscr{A}$ форм областей достиюсимсти есть фактор тора $\mathscr{T}$ по отношению эквивалентности $\mathbf{t}^{\prime} \sim \mathbf{t}^{\prime \prime} \Longleftrightarrow \mathbf{t}^{\prime}= \pm \mathbf{t}^{\prime \prime}$. Если для любого соотношения (10) между частотами $\sum m_{k} \equiv 0 \bmod 2$, то каждый класс эквивалентности на $\mathscr{T}$ состоит из двух элементов, в противном случае - из одного.

В частности, в условиях теоремы аттрактор $\mathscr{A}$ всегда сам является в естественным смысле тором, изогенным тору $\mathscr{T}$. Размерность аттрактора совпадает с рангом группы по сложению, порожденной чисто мнимыми собственными значениями матрицы системы $A$.

\section{ЛитЕРАТУРА}

[1] А. А. Аграчев, Ю. Л. Сачков, Геометрическая теория управления, Физматлит, М., 2004.

[2] F. L. Chernousko, State Estimation of Dynamic Systems, SRC Press, Boca Raton, Florida, USA, 1994.

[3] Е. В. Гончарова, А. И. Овсеевич, Асимптотика множеств достижимости линейных динамических систем с импульсным управлением, Изв. РАН, Теор. сист. упр., 2007, № 1, 51-59.

[4] T. Yu. Figurina, A. I. Ovseevich, Asymptotic behavior of attainable sets of linear periodic control systems, J. Optim. Theory Appl., 100:2 (1999), 349-364.

[5] F. John, Extremum problems with inequalities as subsidiary conditions, in: Studies and Essays Presented to R. Courant on his 60th Birthday, January 8, 1948, Interscience Publishers, New York, NY, 1948, 187-204.

Институт проблем механики РАН e-mail: ovseev@ipmnet.ru
Поступило в редакцию 10 октября 2008 г. 